\title{
Medicinal chemistry at a crossroads: challenges and new possibilities
}

\author{
"It appears that a change of course is needed, one that involves employing much more \\ efficient and logical approaches to improve the success rate of drug discovery \\ and development."
}

Drug discovery and development in the pharmaceutical industry is currently at a crossroads. The precise duration of the current global economic downturn remains to be known, yet before it began in 2007, there had been a clear slowdown in the number of new drug launches announced by major pharmaceutical firms. The slump in productivity can be attributed to numerous factors, including:

- Extensive industry consolidation: in 1988 in the USA, 41 companies were members of the Pharmaceutical Research and Manufacturing Association, whilst that number now stands at 14. The number will further reduce to 12 after the recently announced mergers of Pfizer and Wyeth as well as Merck and Schering-Plough

- Increased number of studies needed for new drug applications in order to demonstrate the safety of medicines

- Increased number of studies needed for new drug applications for drug differentiation from currently available products

Statistics show an unexpectedly high attrition rate $(42 \%)$ for the very expensive late stage of pharmaceutical development (i.e., Phase III clinical trials) for drug candidates during the period of 1990-2002. The primary reasons for the attritions prior to 2002 were a lack of efficacy compared with placebo and unexpected toxicity. Conversely, the major reasons in the last few years have been:

- Little clinical validation of genomics targets

- Inability to demonstrate significant efficacy

- Inability to differentiate from the standard of care

All these challenges have contributed to pushing up the R\&D costs. Figures indicate that launching one US FDA-approved smallmolecule drug or biologic stood at US\$431 million in 1995, US\$660 million in 2000 and US $\$ 1.63$ billion in 2005. This upward trend is expected to continue in the coming years.

How do we respond to these challenges? It appears that a change of course is needed, one that involves employing much more efficient and logical approaches to improve the success rate of drug discovery and development. In order for us to achieve a breakthrough in developing new and efficacious drugs with a minimized attrition rate, we should be able to select patients who will respond to the drugs in a positive manner as well as select patients who will have adverse responses, prior to beginning human clinical trials. Logically, if the preselection of positive responders and adverse responders can be carried out on pharmacogenomic grounds, human clinical trials can be performed quicker and in a more cost-effective manner with a lower attrition rate. This approach could potentially revolutionize the drug-discovery and development process.

\section{"...big pharma have the capital to support extensive and expensive human clinical trials, while biotech and smaller pharma can provide promising experimental drugs to big pharma's pipelines."}

Extensive consolidation of big pharma has promoted the mutual dependence of big pharma on biotechnology as well as small/mediumsized companies: big pharma have the capital to support extensive and expensive human clinical trials, while biotech and smaller pharma can provide promising experimental drugs to big pharma's pipelines. Biotech and smaller

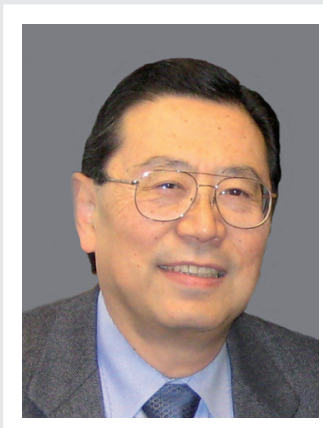

Iwao Ojima

Senior Editor, Future

Medicinal Chemistry,

State University of New York at Stony Brook, Institute of Chemical Biology \& Drug Discovery, Stony Brook, NY II794-3400, USA

E-mail: iojima@ notes.cc.sunysb.edu 
pharma can take up opportunities eschewed by the larger pharmaceutical companies as well as explore more speculative approaches, new medical technologies and hypotheses. In addition, academia is responsible for a great many fundamental discoveries and breakthroughs, supported, in good part, by federal and state funding. Accordingly, industry/academia/government collaborations or consortia have been advancing as major research enterprises.

Under these rapidly changing conditions, medicinal chemistry must play a central role in drug discovery and development through lead discovery and optimization to meet all necessary requirements for drug candidates to become new medicines. However, conventional tools for medicinal chemistry are facing serious challenges. For instance, it has been pointed out that:

- High-throughput screening has not lived up to its original promises

- Combinatorial chemistry did not deliver highquality starting points for lead generation and lead optimization

- Major pharmaceutical firms have large numbers of compound libraries, but these are unsuitable starting points for producing drug-like molecules

What then is the best approach for highly efficient drug discovery? The future of medicinal chemistry is inevitably dependent on the answers to this question. It is, therefore, even more important that the current and the subsequent generations of medicinal chemists are alert and responsive to the rapidly evolving nature of the field.

\section{“...the current and the subsequent generations of medicinal chemists must be alert and responsive to the rapidly evolving nature of the field."}

As an emerging methodology/technology, fragment-based drug discovery is attracting much attention in this post-combinatorial chemistry era. This is partly because it is the next logical step for structure-based drug design. The fast-throughput $\mathrm{x}$-ray crystallography of protein-ligand co-crystals has made this approach practical. The impressive development of computational and structural biology as well as chemical biology strongly supports the revival of structure-based drug design - wherein original medicinal chemistry was born - with new insights. The use of combinatorial chemistry or parallel synthesis for the optimization of highly promising lead compounds arising from the drug-discovery processes around privileged structures and their combinations has been successful in many discovery and development efforts. The focused library approach is especially beneficial for clearing absorption, distribution, metabolism, excretion and toxicology requirements. Consequently, molecular target-based drug discovery has been advancing steadily, with continuous improvements in the quality and diversity of compound libraries.

\section{"...combinatorial biosynthesis and gene mining are emerging as new technologies that may bring about a significant paradigm shift in drug discovery."}

In accordance with this trend, modern natural product chemistry has been re-emerging as a highly promising provider of the sufficiently sophisticated lead structures required for drug discovery. A combination of natural product chemistry and focused library synthesis presents a powerful tool for drug discovery. Also, structural biology, computational biology, chemical biology and chemical genetics are now fully incorporated into the drug discovery pipeline. With these modern tools in hand, it is a natural evolutionary step for chemical genetics to promote structure-based rational drug design, exploring the diverse structures of natural products as well as natural productor drug-like compounds.

In addition, combinatorial biosynthesis and gene mining have the potential to provide fascinating approaches to modern drug discovery. The detailed genetic and biochemical understanding of such biosynthetic pathways will eventually lead to the development of engineered nonribosomal peptide synthetase/ polyketide synthase systems that efficiently generate novel structures with significant therapeutic potential. Also, rapid development in genomics has made quite a large amount of DNA sequence data from a wide variety of organisms available in publicly accessible databases. The gene-mining approach exploits such data to characterize human physiological processes, to identify and validate new drug targets in human pathogens and to discover new chemical entities from natural sources. Thus, 
such new technology has the potential to play a significant role in identifying new bioactive chemical entities and lead compounds. It could be said that combinatorial biosynthesis and gene mining are emerging as technologies capable of bringing about a significant paradigm shift in drug discovery.

The critical contributions of medicinal chemistry to modern drug discovery are not limited to the identification and synthesis of active drug candidates. Medicinal chemistry plays an increasingly important role in the development of efficacious delivery systems, especially for targeted drug delivery. At present, the fields of organic, medicinal, materials and nano-chemistry are involved in such multidisciplinary endeavors. Insights from these allied disciplines have led to and will lead to the invention of new drug conjugates as chemical entities. The use of functional nanomaterials as well as designed polymers as vehicles for drug delivery is another novel approach to pharmaceutical discovery.

By combining new emerging tools with proven traditional ones, molecularly engineered nanobiomaterials could provide unique extracellular matrices for in vitro and in vivo models for human diseases, that are much better than the presently widely used xenograft models. These novel materials, as well as the disease models using them, will make preclinical evaluation of the efficacy and toxicology of drug candidates much more accurate and predictable for use in humans. As such, the development of new animal models relevant to human diseases is critical to significantly reducing the attrition rate and increasing the success rate in drug discovery and development. The future of medicinal chemistry will be inevitably evolving into much more inter- and multidisciplinary endeavours, such as those outlined, a prospect that is truly exciting.

Financial \& competing interests disclosure The author has no relevant affliations or financial involvement with any organization or entity with a financial interest in or financial conflict with the subject matter or materials discussed in the manuscript. This includes employment, consultancies, honoraria, stock ownership or options, expert testimony, grants or patents received or pending, or royalties.

No writing assistance was utilized in the production of this manuscript. 